\title{
Recombinant Murine Granulocyte Macrophage Colony-stimulating Factor Has Megakaryocyte Colony-stimulating Activity and Augments Megakaryocyte Colony Stimulation by Interleukin 3
}

\author{
B. E. Robinson, H. E. McGrath, and P. J. Quesenberry \\ University of Virginia School of Medicine, Charlottesville, Virginia 22908
}

\begin{abstract}
Recombinant murine granulocyte macrophage colony-stimulating factor (rGM-CSF) has been produced in Escherichia coli and purified to homogeneity. GM-CSF has an established role as an in vitro regulator of granulocyte and macrophage colony formation. We have determined that rGM-CSF also has intrinsic activity as a megakaryocyte colony-stimulating factor and that rGM-CSF augments the effect of interleukin 3 (IL-3) on megakaryocyte colony formation. The dose-response curve for megakaryocyte colony induction with rGM-CSF showed plateau megakaryocyte stimulation at $9 \mathrm{ng} / \mathrm{ml}$. When IL-3 (at a plateau dose for megakaryocyte colony induction) was added to rGMCSF over a 0-22-ng/ml dose range, the resultant megakaryocyte colony stimulation approximated the sum of the levels of stimulation produced by either factor alone. These results establish GM-CSF as a multilineage growth factor with definite megakaryocyte colony-stimulating activity and indicate that both GMCSF and IL-3 are important in the regulation of megakaryocytopoiesis.
\end{abstract}

\section{Introduction}

The development of culture techniques that are capable of supporting the growth of rodent and human megakaryocytes and the availability of purified and cloned hemopoietic growth factors has greatly enhanced the understanding of megakaryocytopoiesis.

According to the hypothesis originally suggested by Williams and colleagues $(1,2)$, and subsequently substantiated by Long and colleagues (3), two separate activities are required for the maximal production of recognizable megakaryocyte colonies, a promoter of clonal expansion (megakaryocyte colony-stimulating factor, $\mathrm{CSF}^{1}$ ) and a promoter of maturation and differentiation ("potentiator"). Interleukin 3 (IL-3) has been shown to act as a murine megakaryocyte CSF (4-6) and recently, also shown to have activity as a promoter of megakaryocyte differentiation (7). The potentiator has not yet been fully characterized but is found in mouse lung, bone, and peritoneal conditioned media as well as the macrophage cell line P388D1 conditioned media.

\footnotetext{
Address reprint requests to Dr. Robinson.

Received for publication 17 December 1986.
}

1. Abbreviations used in this paper: $\mathrm{CSF}$, colony-stimulating factor; $\mathrm{GM}$ CSF, granulocyte macrophage CSF; IL-3, interleukin 3; PAGE, polyacrylamide gel electrophoresis; PWM-SCM, pokeweed mitogen spleen conditioned media; rGM-CSF, recombinant GM-CSF.

J. Clin. Invest.

(c) The American Society for Clinical Investigation, Inc.

0021-9738/87/06/1648/05 \$1.00

Volume 79, June 1987, 1648-1652
In vitro and biochemical studies suggest that the potentiator shares many properties with the in vivo thrombopoietic stimulatory factors $(3,8,9)$.

The existent data, however, are in conflict with regard to the potential role of another growth factor in megakaryocytopoiesis, granulocyte-macrophage CSF (GM-CSF). Williams and colleagues suggested that this factor did not stimulate murine megakaryocyte colonies (2). In contrast, we have previously demonstrated that partially purified GM-CSF (derived from concanavalin A [Con A] spleen conditioned media, SCM) had megakaryocyte colony-stimulating activity (4). Furthermore, our data suggested that pokeweed mitogen SCM (PWM-SCM), containing high levels of GM-CSF, potentiated IL-3 stimulated megakaryocyte colony formation of plateau levels of IL-3. It was thus hypothesized that PWM-SCM contained another factor that enhanced megakaryocyte colony formation and this factor was probably GM-CSF. More recently, Metcalf and colleagues have also demonstrated that GM-CSF can stimulate megakaryocyte colonies (10).

In this study, we examine the role of recombinant GM-CSF and purified IL-3 on murine megakaryocyte colony formation and establish rGM-CSF as an in vitro regulator of megakaryocytopoiesis and further demonstrate that rGM-CSF augments the effect of IL-3 on megakaryocyte colony formation.

\section{Methods}

Mice. Female Balb/C (Dominion Laboratories, Dublin, VA), 12-16 wk old, were utilized throughout these studies.

Clonal culture. A single layer soft agar culture technique employing McCoy's 5A media with $2 \mathrm{mM}$ L-glutamine, $16 \mathrm{mg} / \mathrm{ml} \mathrm{L}$-asparagine, 8 $\mu \mathrm{g} / \mathrm{ml} \mathrm{L}$-serine, $1 \mathrm{mM}$ sodium pyruvate, $15 \%$ fetal calf serum (FCS) and $1 \times 10^{-4} \mathrm{mM} 2$-mercaptoethanol was used for cloning murine marrow cells in vitro. Standard stimuli for marrow colony growth included IL3 , generously provided by Dr. J. N. Ihle, purified to homogeneity from WEHI-3 conditioned media as previously described (11). Murine rGMCSF, generously provided by Dr. C.-M. Liang, Biogen Research Corporation, Boston, MA, was produced in Escherichia coli by recombinant techniques (12). The synthesis of GM-CSF was directed by a plasmid containing a gene isolated from the EL-4 cell line. After induction of expression and accumulation of the protein in $E$. coli, the recombinant material was purified to $90 \%$ homogeneity (as analyzed by sodium dodecyl sulfate-polyacrylamide gel electrophoresis, SDS-PAGE) by chaotrope buffer extraction and G-100 Sephadex chromatography in $8 \mathrm{M}$ urea. A second rGM-CSF preparation was generously provided by Dr. J. F. DeLamarter, Biogen Research Corp., Geneva, Switzerland. This material was prepared in the same manner described above with the addition of a final S-200 column separation after several renaturation steps and suspension in Tris $\mathrm{HCl}$. This material produced a single symmetrical peak on reverse-phase high-performance liquid chromatography (HPLC). There was no detectable difference between these two preparations with regard to megakaryocyte stimulation alone or in combination with IL3 ; consequently, data from both preparations has been pooled. 


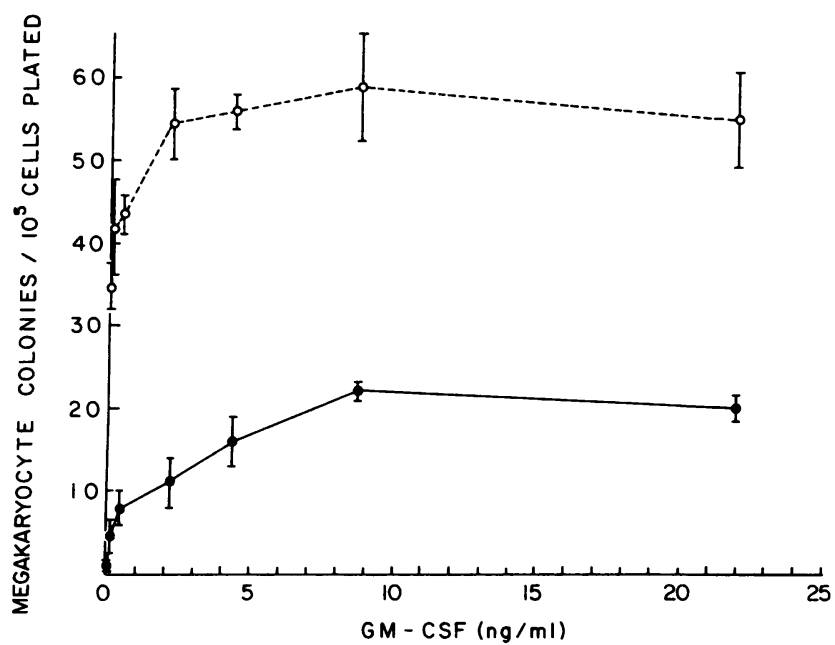

Figure 1. Dose response for megakaryocyte colony formation using GM-CSF alone (๑) and GM-CSF plus IL-3 $(9 \mathrm{ng} / \mathrm{ml})(0)$. Each point represents the mean $( \pm S E M)$ of duplicate plates for each of four separate experiments. (The two curves are significantly different at $P$ $=0.01$.)

Clonal stem cell assays in McCoy's agar were carried out from 50,000 to 100,000 cells $/ \mathrm{ml}$ and colonies were scored after $7 \mathrm{~d}$ of culture. Culture plates were fixed in $10 \%$ formalin and whole agar slide preparations prepared as previously described (13). These slide preparations were stained for acetylcholinesterase and counterstained with hematoxylin (14). Megakaryocyte colonies containing three or more cells were scored. Duplicate plates for each dose level were repeated in four separate experiments.

Statistics. Statistical evaluation was carried out using the Wilcoxon signed rank test (two tails) (15).

Endotoxin. A qualitative limulus lysate assay system (Whittaker MA Bioproducts, Walkersville, MD) was used to determine levels of endotoxin in the rGM-CSF. Correlation between quantitative and qualitative assays has previously established that the qualitative assay can detect endotoxin levels of $0.01 \mathrm{ng} / \mathrm{ml}$. Serial dilutions on the rGM-CSF were used to determine endotoxin levels. E. coli 055:B5 endotoxin (Difco Laboratories, Detroit, MI) was used for the endotoxin addition experiments.

Adherence depletion. Murine bone marrow cells at a concentration of $5 \times 10^{6} / \mathrm{ml}$ in $4 \mathrm{ml} \mathrm{RPMI}+15 \%$ FCS were incubated in $100 \mathrm{~mm}$ polystyrene Corning 25020 tissue culture dishes for $90 \mathrm{~min}$ at $37^{\circ} \mathrm{C}$. The supernatant (nonadherent fraction) was then harvested with a Pasteur pipette. Control marrow was incubated in 50-ml centrifuge tube (Costar 3350, Costar Data Packaging, Cambridge, MA) for $90 \mathrm{~min}$ at $37^{\circ} \mathrm{C}$ and samples were plated simultaneously.

\section{Results}

The dose-response curve for recombinant GM-CSF induction of megakaryocyte colonies is shown in Fig. 1. Plateau megakaryocyte induction occurred at a rGM-CSF dose of $9 \mathrm{ng} / \mathrm{ml}$ that stimulated $22 \pm 1$ colonies $/ 10^{5}$ cells plated. One-half maximal stimulation occurred at a concentration of $2 \mathrm{ng} / \mathrm{ml}$. Media control plates contained $<2$ megakaryocyte colonies $/ 10^{5}$ cells plated.

To determine the effect of the combination of rGM-CSF and IL-3 on megakaryocyte colony formation, we determined the dose response for IL-3, alone, in two separate experiments. Plateau levels of megakaryocyte colony formation occurred at an IL-3 dose of $9 \mathrm{ng} / \mathrm{ml}$ (35 \pm 6 colonies $/ 10^{5}$ cells plated) with one-half maximal induction of $0.5 \mathrm{ng} / \mathrm{ml}$. (Media control plates contained $<2$ colonies $/ 10^{5}$ cells plated.) This one-half maximal induction level was within the range suggested by our previous data on IL-3 induction of megakaryocyte colony formation $(0.14$ to $0.76 \mathrm{ng} / \mathrm{ml}$ ) (4). In four separate experiments, we added 9 $\mathrm{ng} / \mathrm{ml}$ (plateau) IL-3 to $\mathrm{rGM}-\mathrm{CSF}$ over the $0-22 \mathrm{ng} / \mathrm{ml}$ dose range used in the rGM-CSF alone induction experiments. The resultant megakaryocyte stimulation approximates the sum of the levels of stimulation produced by either factor alone as shown in Fig. $1(P=0.01)$. In two similar reciprocal experiments, we found that plateau levels of rGM-CSF $(9 \mathrm{ng} / \mathrm{ml})$ added to IL-3 over a seven point $(0.08-17 \mathrm{ng} / \mathrm{ml})$ dose range also resulted in additive levels of megakaryocyte stimulation at all doses of IL-3 $(P=0.01)$.

Analysis of colony morphology for nonmegakaryocyte colonies $\geq 50$ cells and megakaryocyte colonies $\geq 3$ cells stimulated by rGM-CSF, IL-3, and the combination of both factors at plateau doses for megakaryocyte colony stimulation is shown in Table I. Media control contained low numbers of predominantly "monocytoid" colonies (cells with irregular nuclear outlines and low nuclear to cytoplasmic ratio). The differential counts of colony subtypes stimulated by GM-CSF, alone, showed $54.1 \%$ granulocyte, $22 \%$ macrophage, $12.1 \%$ mixed granulocyte/macrophage, and $11.4 \%$ pure and mixed megakaryocyte colonies. IL-3, alone, stimulated $61 \%$ granulocyte, $17 \%$ macrophage, $5 \%$ mixed granulocyte/macrophage, and $15 \%$ pure and mixed

Table I. Morphologic Analysis of Colonies Stimulated by GM-CSF, IL-3, and GM-CSF + IL-3

\begin{tabular}{|c|c|c|c|c|c|c|c|c|c|}
\hline & \multicolumn{9}{|c|}{ Colonies $/ 10^{5}$ cells plated } \\
\hline & Total & G & $\mathbf{M}$ & $\mathbf{G} / \mathbf{M}$ & Meg & Meg/G & $\mathrm{Meg} / \mathrm{M}$ & $\mathrm{Meg} / \mathrm{G} / \mathrm{M}$ & Mono \\
\hline GM-CSF, 9 ng/ml & $182 \pm 7.2$ & $98.8 \pm 7.2$ & $39.8 \pm 6.4$ & $22 \pm 0.7$ & $20 \pm 1.2$ & $0.3 \pm 0.3$ & 0 & $0.3 \pm 0.3$ & $0.8 \pm 0.5$ \\
\hline $\mathrm{IL}-3,9 \mathrm{ng} / \mathrm{ml}$ & $272 \pm 10.6$ & $165 \pm 17$ & $46.3 \pm 7.2$ & $14 \pm 1.3$ & $33.5 \pm 1.2$ & $0.8 \pm 0.5$ & $1.7 \pm 0.5$ & $5.2 \pm 1.6$ & $5.5 \pm 1.8$ \\
\hline \multicolumn{10}{|l|}{ GM-CSF, $9 \mathrm{ng} / \mathrm{ml}$} \\
\hline$+\mathrm{IL-3}, 9 \mathrm{ng} / \mathrm{ml}$ & $288 \pm 3.4$ & $135 \pm 8.3$ & $60.3 \pm 4.9$ & $26.7 \pm 2.2$ & $49 \pm 2.4$ & $2.2 \pm 0.6$ & $3.5 \pm 1.2$ & $5.8 \pm 2.0$ & $5.5 \pm 1$ \\
\hline Media & $27 \pm 2.3$ & $4 \pm 0.4$ & $3 \pm 0.3$ & 0 & $0.5 \pm 0.3$ & 0 & 0 & 0 & $19.5 \pm 2.5$ \\
\hline
\end{tabular}

G-granulocyte only, M-macrophage only, G/M-mixed granulocyte and macrophage, Meg-megakaryocyte, Meg/G-mixed megakaryocyte and granulocyte, $\mathrm{Meg} / \mathrm{M}$-mixed megakaryocyte and macrophage, $\mathrm{Meg} / \mathrm{G} / \mathrm{M}-$ mixed megakaryocyte, granulocyte, and macrophage, Monomonocytoid cells with irregular nuclear outlines and low nuclear/cytoplasmic ratio. Only colonies containing $\geq 50$ cells were scored except for megakaryocyte colonies where three or more cells were scored. Results represent the mean ( \pm SEM) from four cultures from three separate experiments. Growth factor doses were optimal (plateau) for megakaryocyte colony stimulation. 
Table II. Distribution of Megakaryocyte Colony Sizes Stimulated by GM-CSF, IL-3, and GM-CSF + IL-3

\begin{tabular}{lllll}
\hline \multicolumn{5}{c}{$\begin{array}{l}\text { Number of colonies in each size } \\
\text { class } / 10^{5} \text { cells plated }\end{array}$} \\
\cline { 2 - 5 } Size class (cells/colony) & $3-9$ & $10-19$ & $20-39$ & $>40$ \\
\hline GM-CSF, $9 \mathrm{ng} / \mathrm{ml}$ & $21 \pm 2$ & $1 \pm 0.4$ & 0 & 0 \\
IL-3, $9 \mathrm{ng} / \mathrm{ml}$ & $23 \pm 1$ & $5 \pm 0.9$ & $5 \pm 0.8$ & $1 \pm 0.4$ \\
GM-CSF, $9 \mathrm{ng} / \mathrm{ml}+$ & $39 \pm 3$ & $11 \pm 1.4$ & $6 \pm 1$ & $3 \pm 1$ \\
IL-3, $9 \mathrm{ng} / \mathrm{ml}$ & & & & \\
\hline
\end{tabular}

Results represent the mean ( \pm SEM) for four separate experiments. Growth factor doses were optimal (plateau) for megakaryocyte colony stimulation.

megakaryocyte colonies. The combination of IL-3 and GM-CSF stimulated $47 \%$ granulocyte, $21 \%$ macrophage, $9.2 \%$ mixed granulocyte/macrophage, and $20.9 \%$ pure and mixed megakaryocyte colonies. Each of the stimulated groups also produced small numbers of monocytoid colonies $(<2 \%)$. Although these differential counts suggest that the combination of GM-CSF and IL-3 stimulated greater total numbers of macrophage colonies per $10^{5}$ cells plated than either factor alone, these differences were not significant at the $P<0.05$ level.

More detailed analysis of the sizes of the megakaryocyte colonies stimulated by rGM-CSF, IL-3, and the combination of these two growth factors revealed that, unlike IL-3, rGM-CSF alone did not stimulate the formation of large megakaryocyte colonies containing $>40$ cells. In fact, even at levels of rGMCSF producing maximal numbers of megakaryocyte colonies, colonies which contain more than 10 cells were exceedingly rare (not greater than 1 colony $/ 10^{5}$ cells plated) as shown in Table II. For IL-3, however, the stimulation of large colonies was a dose dependent phenomenon as shown in Table III from two separate experiments. Furthermore, even in cultures stimulated by the combination of GM-CSF and IL-3, the formation of large magakaryocyte colonies ( $\angle 40$ cells) was independent of the concentration of GM-CSF and showed a dose-dependent response to the concentration of IL-3 (data not shown).

Table III. Size of Large Megakaryocyte Colonies Stimulated by $I L-3$

\begin{tabular}{|c|c|c|c|c|}
\hline \multirow[b]{2}{*}{ IL-3 } & \multicolumn{4}{|c|}{ Megakaryocyte colonies $/ 10^{5}$ cells plated } \\
\hline & $\begin{array}{l}\text { Total } \\
\text { ( } \geq 3 \text { cells })\end{array}$ & $\begin{array}{l}\text { Colonies c } \\
\geq 10 \text { cells }\end{array}$ & $\begin{array}{l}\text { 1g: } \\
\geq 20 \text { cells }\end{array}$ & 240 cells \\
\hline \multicolumn{5}{|l|}{$n g / m l$} \\
\hline 17 & $36 \pm 4$ & $13 \pm 4$ & $7 \pm 3$ & $2 \pm 1$ \\
\hline 9 & $36 \pm 4$ & $13 \pm 2$ & $7 \pm 1$ & $3 \pm 0.5$ \\
\hline 4 & $34 \pm 3$ & $12 \pm 1$ & $6 \pm 1$ & $2.3 \pm 0.5$ \\
\hline 2 & $32 \pm 3$ & $11 \pm 1$ & $4 \pm 1$ & $1.3 \pm 0.5$ \\
\hline 0.8 & $29 \pm 6$ & $8 \pm 2$ & $4 \pm 2$ & $1 \pm 0.7$ \\
\hline 0.4 & $24 \pm 2.5$ & $6 \pm 1$ & $3 \pm 0.5$ & $0.5 \pm 0.3$ \\
\hline 0.08 & $10 \pm 3$ & $3 \pm 1$ & 0 & 0 \\
\hline 0 & $1.3 \pm 0.9$ & 0 & 0 & 0 \\
\hline
\end{tabular}

Results represent the mean ( \pm SEM) for four cultures from two separate experiments.
Since rGM-CSF contains detectable levels of endotoxin, we performed a series of experiments designed to evaluate the possibility that contaminating endotoxin was producing the megakaryocyte stimulation. A limulus lysate assay on the rGM-CSF suspended in urea at optimal doses for megakaryocyte colony formation $(9 \mathrm{ng} / \mathrm{ml})$ detected an endotoxin level of $\leq 5 \mathrm{ng} / \mathrm{ml}$. The second preparation suspended in Tris $\mathrm{HCl}$ had endotoxin levels of $\leq 0.14 \mathrm{ng} / \mathrm{ml}$ in the stock solution. In two separate experiments testing the effect of endotoxin on 100,000 murine bone marrow cells, endotoxin over a dose range of $10^{-3} \mathrm{ng} / \mathrm{ml}$ to $1 \mu \mathrm{g} / \mathrm{ml}$ did not stimulate any megakaryocyte colony formation. Similarly, the addition of optimal levels of IL-3 (9 ng/ $\mathrm{ml}$ ) to endotoxin over the same endotoxin dose range showed no further stimulation of megakaryocyte colonies over the level of stimulation produced by IL-3 alone.

Finally, adherent cells have been implicated as accessory cells in megakaryocyte colony formation (16). Two separate experiments revealed that the response to rGM-CSF was actually enhanced by adherence depletion. The results of a representative experiment are shown in Table IV. Preliminary data on more extensive depletions using adherence depletion followed by $\mathbf{B}$ cell depletion (panning with Ig) and finally $\mathrm{T}$ cell depletion (Lyt 1.2 complement lysis depletion) showed that even extensive depletion of macrophages, $B$ and T cells did not abrogate the stimulation of megakaryocyte colonies by rGM-CSF.

\section{Discussion}

It is now clear that many hemopoietic growth factors overlap in their target cell line specificities. For example, in the macrophage lineage, synergy between IL-3 and CSF-1 has been demonstrated (17). Thus, it is not surprising that multifactoral regulation has been demonstrated for megakaryocytopoiesis. IL3 has been well characterized as a potent megakaryocyte CSF (4-6). An, as yet, incompletely characterized megakaryocyte potentiator (differentiation factor) has been described that lacks megakaryocyte CSF activity by itself but synergizes with IL-3 to stimulate megakaryocyte colonies. The potentiator is believed to stimulate acetylcholinesterase incorporation and endomitosis and thus enhance megakaryocyte detection but not to stimulate cell proliferation $(1,8,9)$.

With regard to the role of erythropoietin in megakaryocytopoiesis, the data are contradictory. McLeod and co-workers

Table IV. Comparison of Effects of GM-CSF, IL-3, and GM-CSF $+I L-3$ on Adherence Depleted and Normal Bone Marrow

\begin{tabular}{lll}
\hline & \multicolumn{2}{l}{ Megakaryocyte colonies $/ 10^{5}$ cells plated } \\
\cline { 2 - 3 } & $\begin{array}{l}\text { Adherence } \\
\text { depleted marrow }\end{array}$ & Normal marrow \\
\hline GM-CSF, $9 \mathrm{ng} / \mathrm{ml}$ & 20 & 12.5 \\
$\mathrm{IL}-3,9 \mathrm{ng} / \mathrm{ml}$ & 60.5 & 34 \\
$\mathrm{IL}-3,9 \mathrm{ng} / \mathrm{ml}+\mathrm{GM}-\mathrm{CSF}$, & & \\
$9 \mathrm{ng} / \mathrm{ml}$ & 81.5 & 41.5 \\
Media control & 0.5 & 0 \\
& & \\
\hline
\end{tabular}

Results represent the means of duplicate plates from a single representative experiment. The postadherence cell yield was $46 \%$ of the control group. Viability by trypan blue exclusion for both groups was $>95 \%$. 
(18), in the murine system, and Vainchenker and co-workers (19), in the human system, demonstrated that partially purified human erythropoietin stimulated megakaryocyte colony formation. In contrast, Levin and co-workers (20) observed no effect of partially purified human erythropoietin in their murine megakaryocyte assay. Williams and colleagues (2) found megakaryocyte colony stimulation from one of four batches of partially purified human erythropoietin. More recently, Koike and colleagues (21) demonstrated that purified erythropoietin neither supported megakaryocyte colony formation nor influenced the number of megakaryocytes supported by IL-3. In contrast, Dukes and co-workers (22) have recently demonstrated dose-dependent increases in megakaryocyte colony formation by both recombinant and purified human erythropoietin.

As for the role of GM-CSF in megakaryocytopoiesis, previous work by Williams and colleagues using partially purified GMCSF derived from conditioned media from the lungs of endotoxin treated mice showed no stimulation of megakaryocyte colonies. Further, no enhancement of megakaryocyte colony formation was seen when GM-CSF plus WEHI-3 conditioned media (a source of IL-3) was compared to WEHI-3 alone (2). In contrast, our previous data based on partially purified material from Con A spleen conditioned media suggested that GM-CSF had activity as a megakaryocyte stimulator (4). More recently, Metcalf and co-workers (10) have substantiated our earlier observations using both purified and recombinant GM-CSF demonstrating stimulation of low numbers of megakaryocyte colonies by very high doses of GM-CSF on normal unseparated murine bone marrow. Our current data clearly establish GM-CSF as an in vitro regulator of megakaryocytopoiesis at 10 -fold lower doses than those required in Metcalfs assay system. Plateau dose levels of $9 \mathrm{ng} / \mathrm{ml}$ rGM-CSF stimulated $22 \pm 1$ megakaryocyte colonies (containing three or more cells) per $10^{5}$ cells plated, which was 20 times the level of stimulation seen with media alone. Furthermore, our data demonstrating failure to abrogate the stimulation of megakaryocyte colonies by GM-CSF after adherence, $\mathrm{T}$ - and B-cell depletion of the target bone marrow suggests that GM-CSF has a direct effect on the CFU-megakaryocyte and does not act via an accessory cell population.

As a megakaryocyte CSF, GM-CSF is not as potent as IL3. At plateau levels, GM-CSF induced $\sim 40 \%$ as many megakaryocyte colonies per $10^{5}$ cells plated as IL-3. In addition, GMCSF stimulated megakaryocyte colonies containing fewer total cells than IL-3 and, unlike IL-3, GM-CSF never stimulated colonies containing $>40$ cells in our assay.

Previous work has suggested that endotoxin alone in concentrations of $1-10 \mu \mathrm{g} / \mathrm{ml}$ can stimulate megakaryocyte colony formation (16). In two separate experiments, we found no stimulation of megakaryocyte colonies by endotoxin alone or in combination with IL-3. Thus, the megakaryocyte colony stimulation by GM-CSF cannot be attributed to the presence of contaminating endotoxin.

Further studies will be necessary to understand the mechanism of action and potential interaction of GM-CSF and IL-3 in megakaryocytopoiesis. Interestingly, the level of stimulation achieved by GM-CSF when added to IL-3 approximates the sum of the levels of stimulation produced by either factor alone at all dose levels. This additive result suggests that GM-CSF and IL-3 could be acting on two distinct populations of cells, or, alternatively, acting on the same population, a subset of which requires both regulators for megakaryocyte colony formation.
In conclusion, we have demonstrated that GM-CSF is a multilineage growth factor with definite megakaryocyte colony stimulating activity that likely acts directly on the megakaryocyte progenitor cell. Furthermore, GM-CSF augments megakaryocyte colony formation in the presence of IL-3 indicating that both regulators are important in the control of megakaryocytopoiesis.

\section{Acknowledgments}

We appreciate the assistance of Ms. Wendy Burton for the preparation of the manuscript and Ms. Debbi Lesse for performing the limulus lysate assays.

This work was supported in part by National Institutes of Health grants 5 RO1 AM-27424 and RO1 CA-27466.

\section{References}

1. Williams, N., R. R. Eger, H. M. Jackson, and D. J. Nelson. 1982. Two factor requirements for murine megakaryocyte colony formation. J. Cell. Physiol. 110:101-104.

2. Williams, N., H. Jackson, N. N. Iscove, and P. P. Dukes. 1984. The role of erythropoietin, thrombopoietic stimulating factor, and myeloid colony-stimulating factors on murine megakaryocyte colony formation. Exp. Hematol. 12:734-740.

3. Long, M. W., N. Williams, and T. P. McDonald. 1982. Immature megakaryocytes in the mouse: in vitro relationship to megakaryocyte progenitor cells and mature megakaryocytes. J. Cell. Physiol. 112:339344.

4. Quesenberry, P. J., J. N. Ihle, and E. McGrath. 1985. The effect of interleukin-3 and GM-CSA-2 on megakaryocyte and myeloid clonal colony formation. Blood. 65:214-217.

5. Williams, N., R. Sparrow, K. Gill, D. Yasmeen, and I. McNiece. 1985. Murine megakaryocyte colony stimulating factor: its relationship to interleukin-3. Leuk. Res. 9:1487-1496.

6. Sparrow, R., and N. Williams. 1986. Megakaryocyte colony stimulating factor: its identity to interleukin-3. In Megakaryocyte Development and Function. R. F. Levine, N. Williams, J. Levin, and B. L. Evatt, editors. Alan R. Liss, Inc., New York. 123-128.

7. Ishibashi, T., and S. A. Burstein. 1986. Interleukin-3 promotes the differentiation of isolated single megakaryocytes. Blood. 67:15121514.

8. Williams, N., S. H. Oon, H. Jackson, and R. Lim. 1986. Studies on megakaryocyte potentiator: its production and some biochemical characteristics. In Megakaryocyte Development and Function. R. F. Levine, N. Williams, J. Levin, and B. L. Evatt, editors. Alan R. Liss, Inc., New York. 91-103.

9. Oon, S. H., and N. Williams. 1986. Biochemical characteristics of an in vitro murine megakaryocyte growth activity: megakaryocyte potentiator. Leuk. Res. 10:403-411.

10. Metcalf, D., A. W. Burgess, G. R. Johnson, N. A. Nicola, E. C. Nice, J. Delamarter, D. R. Thatcher, and J. J. Mermod. 1986. In vitro actions on hemopoietic cells of recombinant murine GM-CSF purified after production in Escherichia coli: Comparison with purified native GM-CSF. J. Cell. Physiol. 128:421-431.

11. Ihle, J. N., J. Keller, L. Henderson, F. Klein, and W. Palaszynski. 1982. Procedures for the purification of interleukin-3 to homogeneity. J. Immunol. 129:2431-2436.

12. DeLamarter, J. F., J. J. Mermod, C.-M. Liang, J. F. Eliason, and D. R. Thatcher. 1985. Recombinant murine GM-CSF from $E$. coli has biological activity and is neutralized by a specific antiserum. $E M B O$ (Eur. Mol. Biol. Organ.) J. 4:2575-2581.

13. Gualtieri, R. J., R. K. Shadduck, D. G. Baker, and P. J. Quesenberry. 1984. Hematopoietic regulatory factors produced in long term murine bone marrow cultures and the effect of in vitro irradiation. Blood. 64:516-525. 
14. Jackson, C. W. 1973. Cholinesterase as a possible marker for early cells of the megakaryocytic series. Blood. 42:413-421.

15. Mainland, D. 1963. Elementary Medical Statistics. W. B. Saunders Co., Philadelphia. 273 pp.

16. Williams, N., H. Jackson, P. Ralph, and I. Nakoinz. 1981. Cell interactions influencing murine marrow megakaryocytes. Nature of the potentiator cell in bone marrow. Blood. 57:157-163.

17. McNiece, I., A. B. Kriegler, A. J. Hapel, M. C. Fung, I. G. Young, T. R. Bradley, and G. S. Hodgson. 1984. Recombinant interleukin-3 exhibits synergistic factor activity. Cell. Biol. Int. Rep. 8:811.

18. McLeod, D. L., M. M. Shreeve, and A. A. Axelrod. 1976. Induction of megakaryocyte colonies with platelet formation in vitro. Nature (Lond.). 261:492-494.

19. Vainchenker, W., J. Bouget, J. Gurchard, and J. Breton-Goriun.
1979. Megakaryocyte colony formation from human bone marrow precursors. Blood. 54:940-945.

20. Levin, J., F. C. Levin, D. F. Hall III, and D. G. Penington. 1982. The effect of thrombopoietin on megakaryocyte CFC. With studies of ploidy and platelet size. Blood. 60:989-998.

21. Koike, K., T. Shimizu, T. Miyake, J. N. Ihle, and M. Ogawa. 1986. Hemopoietic colony formation by mouse spleen cells in serum free culture supported by purified erythropoietin and/or interleukin-3. In Megakaryocyte Development and Function. R. F. Levine, N. Williams, J. Levin, and B. L. Evatt, editors. Alan R. Liss, Inc., New York. 33-49.

22. Dukes, P. P., J. C. Egrie, T. W. Strickland, J. K. Broune, and F.-K. Lin. 1986. Megakaryocyte colony stimulating activity of recombinant human and monkey erythropoietin. In Megakaryocyte Development and Function. R. F. Levine, N. Williams, J. Levin, and B. L. Evatt, editors. Alan R. Liss, Inc., New York. 105-109. 